\title{
SERIES SOLUTION METHOD FOR CAUCHY PROBLEMS WITH FRACTIONAL $\triangle$-DERIVATIVE ON TIME SCALES
}

\author{
Svethin G. GEORGIEV AND İNCI M. ERHAN
}

Abstract. In this paper we introduce a series solution method for Cauchy problems associated with Caputo fractional delta derivatives on time scales with delta differentiable graininess function. We also apply the method to Cauchy problems associated with dynamic equations and present some illustrative examples.

Mathematics subject classification (2010): 26A33, 34A08, 34N05, 39A10, 41A58. tion.

Keywords and phrases: Time scale, Caputo fractional delta derivative, series solution, dynamic equa-

\section{REFERENCES}

[1] M. Bohner And A. Peterson, Dynamic Equations on Time Scales: An Introduction with Applications, Birkhäuser, Boston, 2003.

[2] M. Bohner And S. Georgiev, Multivariable Dynamic Calculus on Time Scales, Springer, 2016.

[3] S. Georgiev, Integral Equations on Time Scales, Atlantis Press, 2016.

[4] S. Georgiev, Fractional Dynamic Calculus and Fractional Dynamic Equations on Time Scales, Springer, 2017.

[5] S. Georgiev and I. ERhan, Nonlinear Integral Equations on Time Scales, Nova Science Publishers, 2018

[6] C. Li, Q. YI AND A. CHEN, Finite difference method for nonuniform meshes for nonlinear fractional differential equations, J. Comp. Phys., 316, (2016), 614-631.

[7] M. ORTiguera AND G. Bengochea, A new look at the fractionalization of the logistic equation, Physica A: Statistical Mechanics and its Applications, 467, (2017), 554-561.

[8] J. Quintana-Murillo And S. B. Yuste, A finite difference method with nonuniform timesteps for Fractional diffusion and diffusion-wave equations, Eur. Phys. J. Special Top, 222 8, (2013), $1987-$ 1998.

[9] B. J. West, Exact solution to fractional logistic equation, Physica A: Statistical Mechanics and its Applications, 429, (2015), 103-108. 